\title{
Mechanistic Insights into in vitro DNA Adduction of Oxaliplatin
}

\author{
Yea Seul Kim, ${ }^{a}$ Seonmi Shin, ${ }^{\text {a }}$ Minserk Cheong, and Sang Soo Hah ${ }^{*}$ \\ Department of Chemistry and Research Institute for Basic Sciences, Kyung Hee University, Seoul 130-701, Korea \\ *E-mail:sshah@khu.ac.kr \\ Received April 27, 2010, Accepted May 3, 2010
}

Key Words: Oxaliplatin, DNA adduct, Mechanism of action

(trans- $R, R$ )1,2-Diaminocyclohexaneoxalatoplatinum(II) (oxaliplatin, also known as Eloxatine) is a novel platinum coordination compound, recently approved for the treatment of colorectal cancer, a major cause of cancer deaths worldwide, and has been licensed in the European Union since 1999, and in the United States since 2002 (structure shown in Figure 1). ${ }^{1}$ Oxaliplatin has a broad spectrum of anticancer activity, and importantly, preclinical studies showed that oxaliplatin in combination with 5-fluorouracil has greater in vitro and in vivo anti-proliferative activity than either compound alone in several tumor models, including metastatic colorectal carcinoma. ${ }^{1}$ Interestingly, the cytotoxicity effect of oxaliplatin is not affected in cisplatin-resistant cell lines, providing the first evidence of the absence of cross-resistance between oxaliplatin and cisplatin/carboplatin. ${ }^{2}$

Oxaliplatin has diaminocyclohexane (DACH) as a carrier

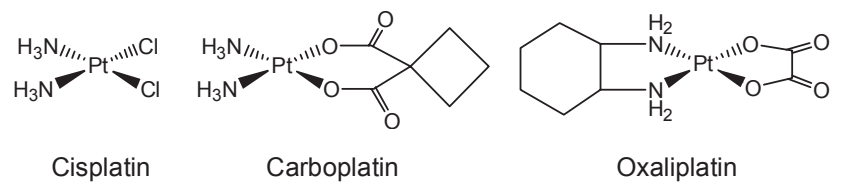

Figure 1. Chemical structures of cisplatin, carboplatin, and oxaliplatin. group and oxalato as a leaving group, and is generally assumed to exert the cytotoxicity effect by interaction with DNA to form monoadducts and intra- and interstrand diadducts, like other platinum-based anticancer drugs such as cisplatin and carboplatin. $^{1,3}$ It has been known that a Pt-DNA level of $\sim 200$ fmol $/ 10 \mu \mathrm{g}$ of DNA reduces cell survival by $90 \%$, and that a Pt-nucleobase binding ratio of $c a$. 1:250,000 is required to initiate apoptosis. ${ }^{4}$

Whereas the mechanisms of action for cisplatin and carboplatin are relatively well established, ${ }^{1,3}$ that for oxaliplatin remains uncertain; there are only a few previous studies on ringopening reactions of oxaliplatin, reporting that the relative unstableness of oxaliplatin to hydrolysis and chloride substitution. ${ }^{5}$ In aqueous solution, it is reported that the oxalate ligand is detached in two steps, forming the oxalate monodentate complex and the dihydrated oxaliplatin complex. ${ }^{5}$ The ring-opening step has a half-life of $16.1 \mathrm{~min}\left(7.17 \times 10^{-4} \mathrm{~s}^{-1}\right)$ and the loss of the oxalate ligand occurs with a half-life of $92.4 \min (1.25 \times$ $\left.10^{-4} \mathrm{~s}^{-1}\right)$ at $37^{\circ} \mathrm{C}$, compared with overall half-lives of $268 \mathrm{~h}$ for carboplatin and $24 \mathrm{~h}$ for cisplatin in chloride-free phosphate buffer at $\mathrm{pH} 7$ and $37^{\circ} \mathrm{C}$. ${ }^{5,6}$ Under in vivo conditions, oxaliplatin is known to undergo extensive biotransformation (see Figure 2 for putative biotransformation pathways of oxaliplatin with low molecular weight endogenous compounds). In plasma ultra-

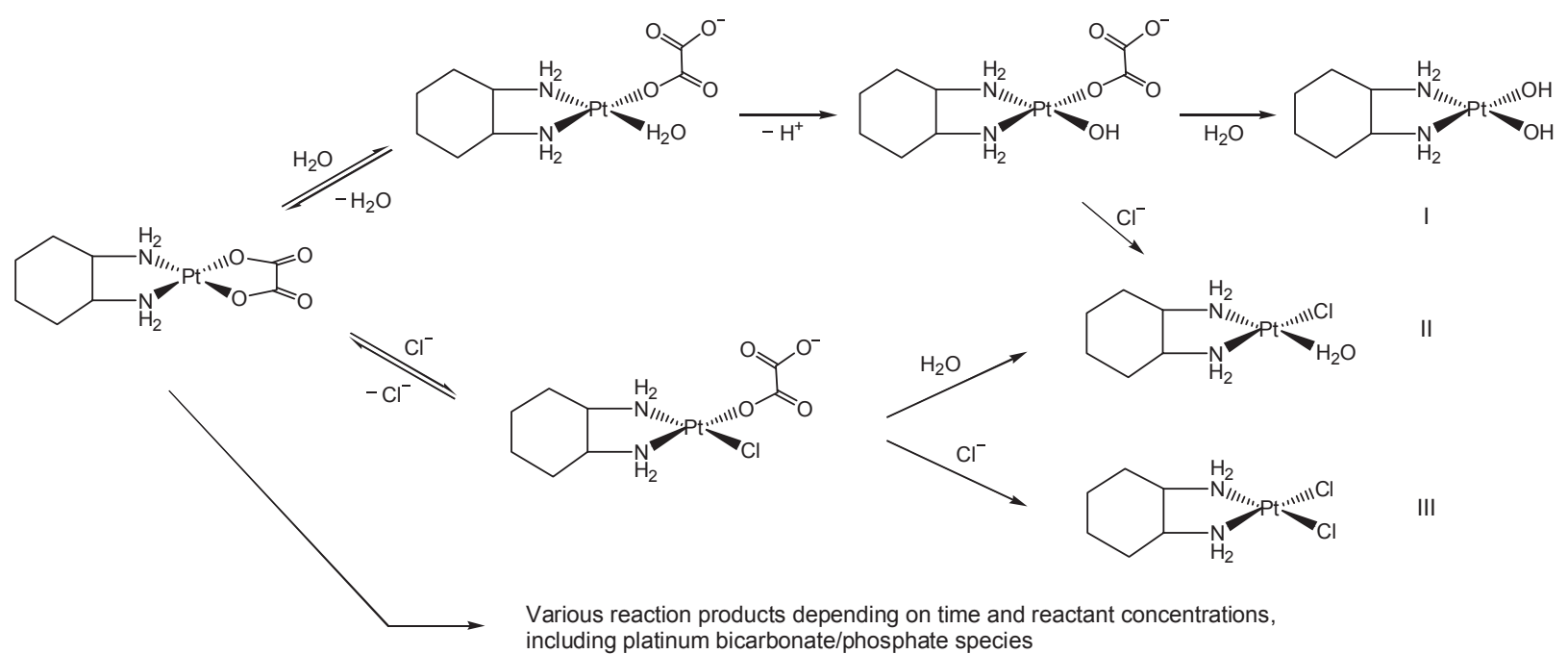

Figure 2. Putative in vivo biotransformation pathways of oxaliplatin with low molecular weight endogenous compounds.

${ }^{\mathrm{a}}$ These authors contributed equally to this work. 
filtrate from cancer patients, for example, biotransformation products with chloride, methionine and glutathione have been identified. $^{7}$

We recently reported the use of an extremely sensitive detection method of accelerator mass spectrometry (AMS), ${ }^{8}$ to study the kinetics of oxaliplatin-DNA adduct formation in DNA. ${ }^{9}$ In this study, we describe a mechanistic refinement of the kinetics of hydration of the parent compound and subsequent covalent binding to DNA.

Salmon sperm DNA was incubated with $\left[{ }^{14} \mathrm{C}\right]$ oxaliplatin over time, and the fraction of the drug bound to the DNA in the form of both monoadducts and diadducts was experimentally determined and plotted as a function of time (Figure 3). The concentrations of oxaliplatin-DNA mono- and diadducts during the time course of the reaction allowed kinetic calculation of oxaliplatin binding to DNA. The radiocarbon content of the DNA displayed an exponential increase over the time course of the experiment, leading to determination of a kinetic constant $k_{\text {obs }}$ to be $3.36 \times 10^{-6} \mathrm{~s}^{-1}\left(\mathrm{R}^{2}=0.989\right)$ by linear regression analysis. $^{6(a)}$ Our results show that the reaction of oxaliplatin with DNA is very slow in vitro, suggesting that the DNA adduct formation in vivo may instead be forming with non-enzymatic biotransformation products of oxaliplatin (Figure 2). We attribute this kinetic profile to parameters defining the rate of oxaliplatin-DNA mono- and diadduct formation and by depletion

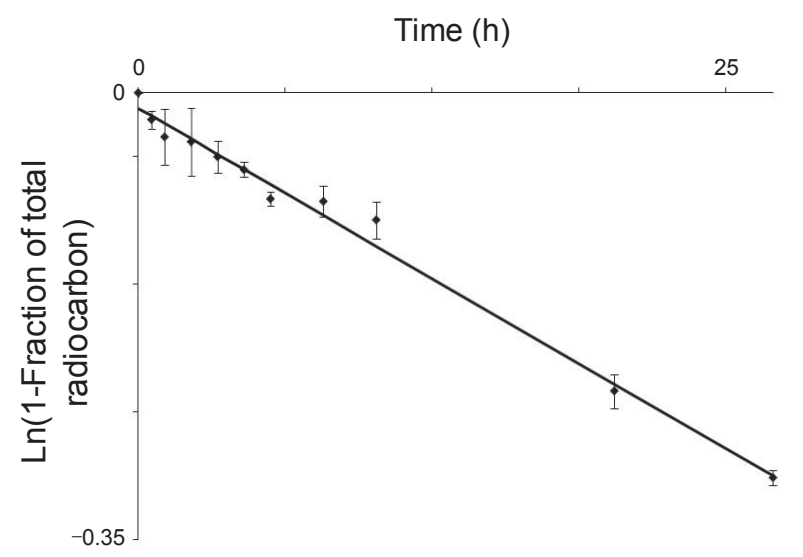

Figure 3. $\operatorname{Ln}$ (1-fraction of total radiocarbon) vs. time plot from experimental and theoretical data for in vitro reaction of oxaliplatin with DNA. The radiocarbon measured by accelerator mass spectrometry (AMS) due to oxaliplatin-DNA mono- and diadducts as a fraction of the total radiocarbon, represented by the symbol $\bullet$. The line was fitted using linear regression analysis with $k_{\mathrm{obs}}=3.36 \times 10^{-6} \mathrm{~s}^{-1}$ (solid line). These data were from our previously published paper (Ref. 9). of the solution of oxaliplatin derivatives capable of reacting with DNA over time.

As depicted in Figure 4, the dominant mechanistic steps of oxaliplatin reacting with DNA likely include rate constants for: mono- and diaquation of the parent compound, $k_{1}$ and $k_{2}$, respectively, formation of oxaliplatin-DNA monoadducts at purine nucleotides, $k_{3}$, and diadduct formation, $k_{4}$. It should be pointed out that in this mechanism chlorination reaction, backward reaction of the first ring-opening step, and acid dissociation of the oxalate monodentate complex are ignored in order to simply understand the mechanism of action for oxaliplatin in the reaction system employed, since 1) the buffer system employed is similar to one $(50 \mathrm{mM} \mathrm{NaCl}, 10 \mathrm{mM}$ HEPES buffer at $\mathrm{pH} 7.4$ and $37^{\circ} \mathrm{C}$ ) in which oxaliplatin degradation was characterized to occur at a few percent per hour to form chlorinated species, although this chlorination reaction should not be ignored under in vivo conditions, ${ }^{10} 2$ ) the series reaction of the hydrolysis of oxaliplatin is known to obey pseudo-first order kinetics and rate of the formation of diaquated species is very fast compared to that of the formation of diaquated cisplatin or carboplatin species (it should be noted, however, that the reverse reaction of oxaliplatin formation is fast when the oxalate monodentate complex is acidified, although at $\mathrm{pH} \geq 12$ the conversion of the oxalate monodentate complex back to oxaliplatin is less than $1 \%),{ }^{5(\mathrm{c})}$ and 3 ) the acid dissociation constant for the oxalate monodentate complex $\left(\sim 5.9 \times 10^{-8} \mathrm{~s}^{-1}\right)$ is approximately 1000 fold smaller than that for formation of diaquated oxaliplatin from the oxalate monodentate complex. ${ }^{5(\mathrm{~b})}$ Moreover, it has been suggested that in order to react with DNA oxaliplatin should be activated in vivo to the diaquated oxaliplatin complex via either of two routes: by reaction with bicarbonate or phosphate followed by hydrolysis giving diaquated oxaliplatin complex or by reaction with chloride giving a dichloro complex which is subsequently hydrolysed to the diaquated species. ${ }^{7}$

An alternative mechanism could involve direct covalent binding of the monoaquated species to DNA, since the formation of monoadduct from the monoaquated oxaliplatin is likely to be kinetically important and the steady state concentration of this species can be greater than the steady state concentration of the diaquated oxaliplatin. However, we observed a substantial amount of non-covalently bound compound, possibly the diaquated +2 charged species, on the DNA especially at early time points, which could be removed by exposure of the solution to concentrated $\mathrm{NaCl}$ prior to DNA isolation (data not shown). In addition, rate of the formation of diaquated oxaliplatin is known to be very fast ${ }^{5(\mathrm{c})}$ and our data cannot be explained without an assumption that a ring-opened monoaquated oxali-

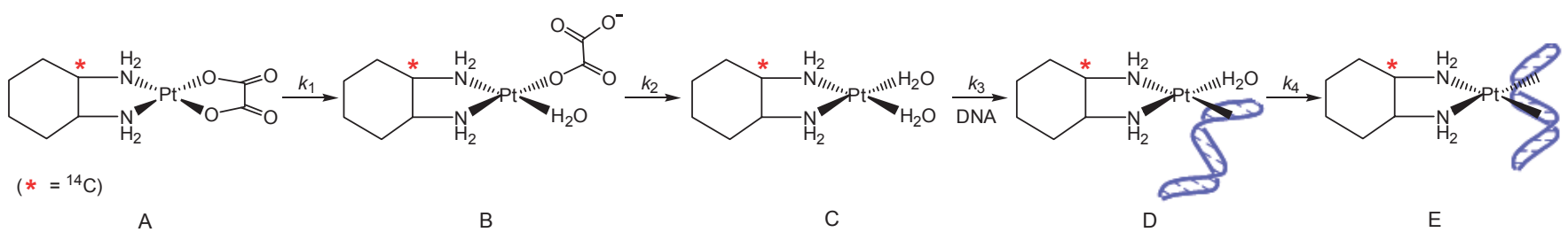

Figure 4. Proposed mechanism of action for oxaliplatin under the conditions in the study (the location of the ${ }^{14} \mathrm{C}$ atom is asterisked). Based on the experimental data, a four consecutive irreversible pseudo-first-order reaction mechanism is assumed to be the major contributor for oxaliplatinDNA monoadduct formation, followed by conversion to diadducts. 
Table 1. Comparison of apparent rate constants for cisplatin and oxaliplatin adduction to DNA. Rate constants $k_{1}, k_{2}, k_{3}$ and $k_{4}$ for cisplatin stand for the rates for monoaquation of cisplatin, monoadduct formation of monoaquated species, monoaquated species of monoadducts, and monoadduct-to-diadduct conversion, respectively, and rate constants for oxaliplatin stand for the rates for monoaquation of the parent compound, diaquation of monoaquated species, monoadduct formation of diaquated species, and monoadduct-to-diadduct conversion, respectively.

\begin{tabular}{lcc}
\hline & cisplatin $^{a}$ & oxaliplatin \\
\hline$k_{1}$ & 0.000102 & $0.000717^{b}$ \\
$k_{2}$ & 0.00196 & $0.000125^{b}$ \\
$k_{3}$ & 0.000092 & $\sim 0.00000576^{c}$ \\
$k_{4}$ & n.d. & $\sim 0.0000438^{d}$ \\
\hline
\end{tabular}

The unit for the kinetics constants is $\mathrm{s}^{-1}$ and $n . d$. represents not determined. ${ }^{a}$ Ref. $11,{ }^{b}$ Ref. 5(c), ${ }^{c}$ In this study, ${ }^{d}$ Ref. 13 , respectively.

platin derivative minimally or negligibly contributes to the oxaliplatin-DNA adduct formation, and thus that the four consecutive irreversible pseudo-first-order mechanism is a major contributor to oxaliplatin-DNA adduct formation. This interpretation is consistent with the presence of a substantial fraction of parent and monoaquated compound early in the reaction, which are quantitatively converted to diaquated $\mathrm{Pt}(\mathrm{DACH})$ prior to covalent binding to DNA. From this point of view, oxaliplatin, like cisplatin, but differently from carboplatin, could be considered as a pro-drug, with hydrolysis being a key step. In fact, hydrolysis of cisplatin or oxaliplatin is extremely rapid, whereas it is slower for carboplatin. $1,3,5$

Based upon the reported apparent rate constant values of $k_{1}$ $\left(7.17 \times 10^{-4} \mathrm{~s}^{-1}\right)$ and $k_{2}\left(1.25 \times 10^{-5} \mathrm{~s}^{-1}\right),{ }^{5}$ we estimated $k_{3}$ to be $(5.76 \pm 0.57) \times 10^{-6} \mathrm{~s}^{-1}$ (see Experimental Section for details). This value is approximately three-hundred-fold lower than reported for cisplatin $\left(k_{2}, 1.96 \times 10^{-3} \mathrm{~s}^{-1}\right.$, Table 1$){ }^{11}$ explaining the lower reactivity of oxaliplatin than cisplatin in the literature. ${ }^{12}$ The kinetic constant $k_{4}$, however, could not be determined with our experimental data, since our method does not distinguish monoadducts from diadducts, although it may be assumed that $k_{4}$ for oxaliplatin monoadduct-to-diadduct conversion is likely close to that reported for diaminocyclohexanedichloroplatinum(II) $\left[\mathrm{PtCl}_{2}(\mathrm{DACH})\right]$ in the literature $\left(4.38 \times 10^{-5} \mathrm{~s}^{-1}\right){ }^{13}$ since $\mathrm{PtCl}_{2}(\mathrm{DACH})$ should also form dihydrated oxaliplatin species prior to DNA adduction.

In conclusion, we refined the kinetics of oxaliplatin-DNA adduct formation, which may allow us to gain new mechanistic insights into in vitro DNA adduction of oxaliplatin, suggesting a mechanism of action for oxaliplatin in vitro (Figure 4), in which the leaving group is replaced by two water molecules in two consecutive steps forming the diaquated oxaliplatin complex, followed by the reaction with DNA to form monoadducts and by conversion to crosslinks. Table 1 summarizes the apparent rate constants for cisplatin- and oxaliplatin-DNA adduct formation in vitro, showing that the in vitro reaction of oxaliplatin with DNA is very slow compared to cisplatin, even if these values for cisplatin and oxaliplatin may not be directly compared. Overall, our kinetic study suggests that the DNA adduct formation of oxaliplatin in vivo may be forming with nonenzymatic biotransformation products of oxaliplatin, support- ing the assumption that in vivo, oxaliplatin undergoes extensive nonenzymatic biotransformation and nucleophiles such as an endogenous bicarbonate and dihydrogen phosphate may displace the oxalate group forming unstable reactants which can be further hydrolyzed to aqua complexes, and that the aqua complexes are likely important for the cytotoxic effect of oxaliplatin, mediated by the formation of DNA adducts.

\section{Experimental Section}

To refine the kinetics of oxaliplatin-DNA adduct formation, we used the experimental data for in vitro DNA binding kinetics, previously described for oxaliplatin studies. ${ }^{9}$ Briefly, a mixture of $\left[{ }^{14} \mathrm{C}\right]$ oxaliplatin (GE Healthcare, Piscataway, NJ) and non-labeled oxaliplatin was prepared immediately prior to use and incubated with salmon sperm DNA $(0.5 \mathrm{mg} / \mathrm{mL})$ at $37{ }^{\circ} \mathrm{C}$ in $25 \mathrm{mM} \mathrm{NaCl}, 0.14 \mathrm{mM}$ EDTA, $0.14 \mathrm{mM}$ Tris $\cdot \mathrm{HCl}$, $\mathrm{pH}$ 7.4. This buffer system is similar to one $(50 \mathrm{mM} \mathrm{NaCl}, 10$ mM HEPES buffer at $\mathrm{pH} 7.4$ and $37^{\circ} \mathrm{C}$ ) in which oxaliplatin degradation was characterized to occur at a few percent per hour to form chlorinated species. ${ }^{10}$ The initial concentration of oxaliplatin was $12.4 \mu \mathrm{g} / \mathrm{mL}(33.4 \mathrm{nmol}, 33.4 \mu \mathrm{M})$, which contained $13.4 \mathrm{dpm}(96.8 \mathrm{fmol})$ of $\left[{ }^{14} \mathrm{C}\right]$ oxaliplatin $(77.6 \mu \mathrm{Ci}$ / mmol). Samples $(200 \mu \mathrm{L})$ of the solution were taken at 11 time points up to $24 \mathrm{~h}$ (see Figure 3 for specific time points sampled), and were immediately adjusted to $0.5 \mathrm{M} \mathrm{NaCl}$ and frozen in liquid nitrogen. For AMS sample preparation, the samples were thawed on ice and the DNA was isolated at $4{ }^{\circ} \mathrm{C}$ by ethanol precipitation and air dried. The dried samples were redissolved in medium containing $50 \mathrm{mM}$ sodium acetate, $10 \mathrm{mM} \mathrm{MgCl}_{2}$, $\mathrm{pH} 5.5$, as described for ethanol precipitation. ${ }^{14}$ Variance in DNA recovery, which ranged from 10 - 70 percent, did not influence the radiocarbon concentrations in the resulting AMS samples as evidenced by the high precision of the AMS measurements from triplicate experiments. The DNA was then converted to graphite, and analyzed for the ratio of ${ }^{14} \mathrm{C}$ to total carbon by AMS, as previously described. ${ }^{3(a), 9}$ All experiments were carried out in triplicate.

Kinetic calculation. When focusing on the carrier group (diaminocyclohexane or DACH) of oxaliplatin, there are at least five predominant forms of oxaliplatin derivatives in the reaction solution: Unreacted oxaliplatin (A), monoaquated oxaliplatin (B), diaquated oxaliplatin (C), monofunctional oxaliplatinDNA adduct (D), and difunctional oxaliplatin-DNA adduct (E). And because the concentration of the target sites in DNA is highly excess, the reaction kinetics can be assumed as the pseudo-first order (i.e. the concentration of the DNA target remains in constant vast excess), allowing for the determination of $k_{\text {obs }}$. The chlorination reaction of oxaliplatin is known to be negligibly slow, ${ }^{10}$ and the corresponding forms have been ignored in terms of kinetic consideration in this study, leading to a four-step consecutive irreversible pseudo-first-order reaction scheme. The overall rate constant $k_{\text {obs }}$ can be used to calculate each rate constant based on the equation of $1 / k_{\mathrm{obs}}=1 / k_{1}+1 / k_{2}+$ $1 / k_{3}+1 / k_{4}$, which can be used to estimate the rate constant $k_{3}$, when other rate constants $k_{1}, k_{2}$ and $k_{4}$ are known.

Alternatively, $k_{3}$ can be estimated using the following equations where $\mathrm{A}_{0}$ is the starting concentration of oxaliplatin, based 
on the assumption of a four-step consecutive irreversible mechanism and the reported values of $k_{1}, k_{2}$ and $k_{4}{ }^{3(\mathrm{a})}$

$$
\begin{aligned}
{[\mathrm{A}]=} & \mathrm{A}_{0} e^{-k_{1} \mathrm{t}} \\
{[\mathrm{B}]=} & \frac{k_{1} \mathrm{~A}_{0}}{k_{2}-k_{1}}\left[e^{-k_{1} \mathrm{t}}-e^{-k_{2} \mathrm{t}}\right] \\
{[\mathrm{C}]=} & k_{1} k_{2} \mathrm{~A}_{0}\left[e^{-k_{1} \mathrm{t}} /\left\{\left(k_{2}-k_{1}\right)\left(k_{3}-k_{1}\right)\right\}+e^{-k_{2} \mathrm{t}} /\left\{\left(k_{1}-k_{2}\right)\left(k_{3}-k_{2}\right)\right\}\right. \\
& \left.+e^{-k_{3} \mathrm{t}} /\left\{\left(k_{1}-k_{3}\right)\left(k_{2}-k_{3}\right)\right\}\right] \\
{[\mathrm{D}]=} & k_{1} k_{2} k_{3} \mathrm{~A}_{0}\left[e^{-k_{1} \mathrm{t}} /\left\{\left(k_{4}-k_{1}\right)\left(k_{3}-k_{1}\right)\left(k_{2}-k_{1}\right)\right\}\right. \\
& +e^{-k_{2} \mathrm{t}} /\left\{\left(k_{4}-k_{2}\right)\left(k_{3}-k_{2}\right)\left(k_{1}-k_{2}\right)\right\} \\
& +e^{-k_{3} \mathrm{t} t} /\left\{\left(k_{4}-k_{3}\right)\left(k_{2}-k_{3}\right)\left(k_{1}-k_{3}\right)\right\} \\
& \left.+e^{-k_{4} \mathrm{t}} /\left\{\left(k_{3}-k_{4}\right)\left(k_{2}-k_{4}\right)\left(k_{1}-k_{4}\right)\right\}\right] \\
{[\mathrm{E}]=} & \mathrm{A}_{0}-[\mathrm{A}]-[\mathrm{B}]-[\mathrm{C}]-[\mathrm{D}]
\end{aligned}
$$

Acknowledgments. Drs. Paul T. Henderson and Kenneth W. Turteltaub are acknowledged for helpful advice. This work was supported by Basic Science Program through the National Research Foundation of Korea (KRF) funded by the Ministry of Education, Science and Technology (MEST) (No. 20100015218)

\section{References}

1. (a) Saunders, M.; Iveson, T. Br. J. Cancer 2006, 95, 131-138. (b) Graham, J.; Muhsin, M.; Kirkpatrick, P. Nat. Rev. Drug Discov. 2004, 3, 11-12. (c) Wang, D.; Lippard, S. J. Nature Rev. Drug Discov. 2005, 4, 307-320. (d) Jamieson, E. R.; Lippard, S. J. Chem. Rev. 1999, 99, 2467-2498.

2. (a) Pendyala, L.; Creaven, P. J. Cancer Res. 1993, 53, 5970-5976. (b) Di Francesco, A. M.; Ruggiero, A.; Riccardi, R. Cell Mol. Life Sci. 2002, 59, 1914-1927.

3. (a) Hah, S. S.; Stivers, K. M.; de Vere White, R. W.; Henderson, P. T. Chem. Res. Toxicol. 2006, 19, 622-626. (b) Chaney, S. G.; Campbell, S. L.; Bassett, E.; Wu, Y. Crit. Rev. Oncol. Hematol. 2005, 53, 3-11. (c) Desoize, B.; Madoulet, C. Crit. Rev. Oncol. Hematol. 2002, 42, 317-325. (d) Kartalou, M.; Essigmann, J. M. Mutat. Res. 2001, 478, 1-21. (e) Fuertes, M. A.; Alonso, C.; Perez, J. M. Chem. Rev. 2003, 103, 645-662.

4. Oshita, F.; Eastman, A. Oncol. Res. 1993, 5, 111-118.

5. (a) Summa, N.; Schiessl, W.; Puchta, R.; van Eikema, H. N.; van Eldik, R. Inorg. Chem. 2006, 45, 2948-2959. (b) Jerremalm, E.; Eksborg, S.; Ehrsson, H. J. Pharm. Sci. 2003, 92, 436-438. (c) Jerremalm, E.; Videhult, P.; Alvelius, G.; Griffiths, W. J.; Bergman, T.; Eksborg, S.; Ehrsson, H. J. Pharm. Sci. 2002, 91, $2116-$ 2121.

6. (a) Knox, R. J.; Friedlos, F.; Lydall, D. A.; Roberts, J. J. Cancer Res. 1986, 46, 1972-1979. (b) Canovese, L.; Cattalini, L.; Chessa, G.; Tobe, M. L. J. Chem. Soc., Dalton Trans. 1988, 8, 2135-2140.

7. Graham, M. A.; Lockwood, G. F.; Greenslade, D.; Brienza, S.; Bayssas, M.; Gamelin, E. Clin. Cancer Res. 2000, 6, 1205-1218.

8. (a) Hah, S. S. J. Biomed. Sci. 2009, 16, 54. (b) Hah, S. S. Anal. Sci. 2009, 25, 731-733. (c) Mundt, J. M.; Hah, S. S.; Sumbad, R. A.; Schramm, V.; Henderson, P. T. Nucleic Acids Res. 2008, 36, 228-236. (d) Hah, S. S.; Mundt, J. M.; Ubick, E. A.; Turteltaub, K. W.; Gregg, J. P.; Henderson, P. T. Nucl. Instr. Methods Phys. Res. B 2007, 259, 763-766. (e) Hah, S. S.; Kim, H. M.; Sumbad, R. A.; Henderson, P. T. Bioorg. Med. Chem. Lett. 2005, 15, 36273631.

9. Hah, S. S.; Sumbad, R. A.; Vere White, R. W.; Turteltaub, K. W.; Henderson, P. T. Chem. Res. Toxicol. 2007, 20, 1745-1751.

10. Jerremalm, E.; Hedeland, M.; Wallin, I.; Bondesson, U.; Ehrsson, H. Pharm. Res. 2004, 21, 891-894.

11. Bancroft, D. P.; Lepre, C. A.; Lippard, S. J. J. Am. Chem. Soc. 1990, 112, 6860-6871.

12. Woynarowski, J. M.; Chapman, W. G.; Napier, C.; Herzig, M. C. S.; Juniewicz, P. Mol. Pharmacol. 1998, 54, 770-777.

13. Page, J. D.; Husain, I.; Sancar, A.; Chaney, S. G. Biochemistry 1990, 29, 1016-1024.

14. Jennerwein, M. M.; Eastman, A.; Khokhar, A. Chem. Biol. Interact. 1989, 70, 39-49. 